\title{
Dynamic centrifuge testing to assess liquefaction potential
}

\author{
G. Fasano, E. Bilotta and A. Flora \\ University of Napoli Federico II, Italy \\ V. Fioravante and D. Giretti \\ University of Ferrara, ISMGEO, Seriate (BG), Italy \\ C.G. Lai and A.G. Özcebe \\ University of Pavia, Italy
}

\begin{abstract}
A set of centrifuge tests has been carried out at ISMGEO (Italy) laboratory on models of a liquefiable soil. A natural sand from the Emilia-Romagna region in Italy was used in the tests, in order to reproduce typical ground conditions where liquefaction occurred during the seismic sequence of 2012. The models were instrumented with miniaturised accelerometers and with pore pressure and displacement transducers. Spectrum-compatible acceleration time histories were applied at the base of the model. In this way triggering of the liquefaction was detected and post-liquefaction settlements were evaluated. The paper describes with the tests carried out on free-field models. Further tests are currently ongoing to assess the seismic response of simple model structures lying on liquefiable ground. The testing programme, funded within the H2020 research project LIQUEFACT, is aimed at an experimental verification of ground improvement techniques used to mitigate the liquefaction susceptibility of fully saturated loose sands.
\end{abstract}

\section{INTRODUCTION}

In seismic regions, soil liquefaction has been often one of the most significant causes of damage to structures during recent earthquakes, e.g. 2012 Emilia (northern Italy), 2011 Tohoku Oki (Japan) and particularly 2010-2011 Canterbury-Christchurch (New Zealand), where about half of the $€ 25$ billion loss was directly caused by soil liquefaction. Earthquake-induced liquefaction disasters have now been included among the most important induced effects of natural hazards on human environment. Enhancement of liquefaction risk assessment is needed to protect life and public safety and to mitigate economic, environmental, and societal impacts of liquefaction in a cost-effective manner (National Academies of Sciences, Engineering, and Medicine, 2016). In this framework physical modelling assumes relevance since it allows a number of different scenarios of hazard to be analysed in well-defined geotechnical conditions.

This work focuses on centrifuge tests carried out on free-field models of liquefiable ground, as part of a larger experimental campaign: further tests on models including simple structures are currently underway. The experimental programme, funded within the H2020 research project LIQUEFACT, is aimed at verifying the effectiveness of a number of ground improvement techniques to mitigate the liquefaction susceptibility of fully saturated loose sands.

\section{BACKGROUND}

Liquefaction under cyclic loading may occur in saturated soil, due to the increase of pore-water pressure. It consists in a significant loss of shear strength and stiffness of the soil that may result in instability of existing structures or trigger settlement, tilting or lateral spreading. The main aspects of the mechanics of soil liquefaction have been traditionally investigated via laboratory tests, study of case histories and physical modelling. It is worth mentioning the validation exercise VELACS (Verification of Liquefaction Analyses by Centrifuge Studies), that was carried out about 25 years ago by a number of centrifuge laboratories in UK and US. The experimental results showed significant variability. Nevertheless, they allowed a fruitful comparison of numerical blind predictions by several teams. The results indicated that numerical modelling at that time was quite challenging due to boundary problems involving soil liquefaction (Manzari et al., 2014).

More recently, the LEAP project (Liquefaction Experiments and Analysis Project) has been launched to produce a set of high quality centrifuge test data that will be used to assess the capabilities of currently available computational tools (Zeghal et al., 2014).

Nowadays it is largely accepted that a combined use of physical and numerical modelling is necessary to predict the effect of earthquake-induced liquefaction on buildings and other subsystems of 
civil infrastructures. The experimental activity carried out within the LIQUEFACT project follows this stream.

\section{EXPERIMENTAL PROGRAMME}

The set of centrifuge tests that will be shortly completed within LIQUEFACT is currently underway at ISMGEO (Italy) laboratory to assess the effectiveness of two selected ground treatment techniques against liquefaction: drainage and induced partial saturation (IPS).

ISMGEO centrifuge has an arm of $3 \mathrm{~m}$ and a capacity of $240 \mathrm{~g}$-tonnes (maximum payload $400 \mathrm{~kg}$, max acceleration $600 \mathrm{~g}$ ). In 2010 it was equipped with a 1 degree-of-freedom shaking table. Two hydraulic actuators fire input signals up to $1 \mathrm{MHz}$ at $100 \mathrm{~g}$. The peak velocity of the shaking table is $0.9 \mathrm{~m} / \mathrm{s}$ and the peak displacement $6.35 \mathrm{~mm}$.

The LIQUEFACT testing programme includes free-field modelling to compare the response of untreated and treated ground. This paper refers only to this subset of tests on two untreated models.

\subsection{Material}

A natural sand from the Emilia-Romagna region in Italy was used in the tests to reproduce typical ground conditions where liquefaction occurred during the2012 seismic sequence. The sand was retrieved from the field trial site of the project. This is located along the Reno river close to the town of Pieve di Cento near Bologna. This is a silica sand $\left(\mathrm{G}_{\mathrm{S}}=2.69\right.$, $\gamma_{\mathrm{d}, \min }=12.25 \mathrm{kN} / \mathrm{m}^{3}, \quad \gamma_{\mathrm{d}, \max }=15.75 \mathrm{kN} / \mathrm{m}^{3}$ ) with a coefficient of uniformity $\mathrm{U} \cong 1.8$ (Figure 1 ). The sand has been characterised under both static and cyclic loading conditions (Mele et al., unpubl.).

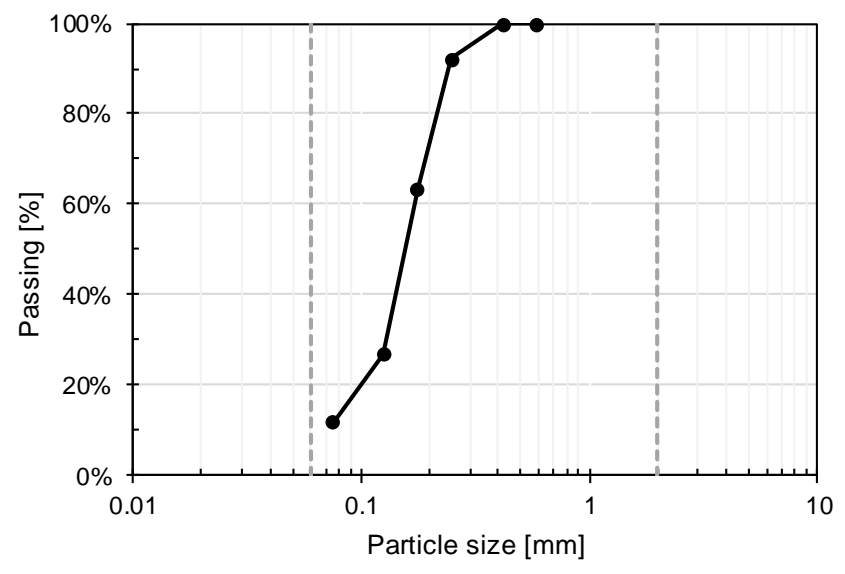

Figure 1. Grain size distribution of Pieve di Cento sand.

Model M1_S2 was made of clean Pieve di Cento sand, that is, after eliminating the finest fraction, passing at the ASTM no.200 sieve. Model M1_S3 was made using Pieve di Cento sand as it is (Fig. 1).

\subsection{Centrifuge container}

An ESB (Equivalent Shear Beam, Zeng and Schofield, 1996) container has been purposely manufactured by ISMGEO laboratory to be used during dynamic tests (Figure 2). This consists of 12 rectangular aluminum frames, each $25 \mathrm{~mm}$ thick, separated by 11 rubber layers (shear modulus, $\mathrm{G}_{\mathrm{r}, 0}$ $=1.3 \mathrm{MPa}$; tensile strength $\sigma_{\mathrm{f}}=4 \mathrm{MPa}$, tensile strain at failure, $\varepsilon_{\mathrm{f}}=250 \%$ ), each $3.36 \mathrm{~mm}$ thick. The whole height of the container is $337 \mathrm{~mm}$. The main characteristics of the container are shown in Table 1.
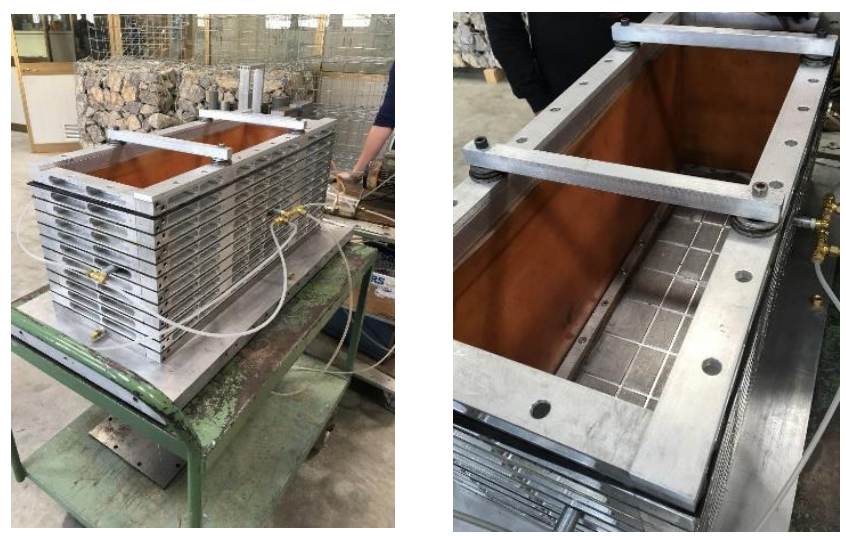

Figure 2. ESB container at ISMGEO laboratory.

\begin{tabular}{cc} 
Table 1. Characteristics of ISMGEO ESB container \\
\hline Number of frames (-) & 12 \\
Number of rubber layers (-) & 11 \\
Frame mass $(\mathrm{kg})$ & 3.4 \\
Height $(\mathrm{mm})$ & 337 \\
Internal width $(\mathrm{mm})$ & 250 \\
Internal length $(\mathrm{mm})$ & 750 \\
Frame thickness $(\mathrm{mm})$ & 25 \\
Frame width $(\mathrm{mm})$ & 40 \\
Rubber layer thickness $(\mathrm{mm})$ & 3.36 \\
Container weight $(\mathrm{kg})$ & 110 (incl. base) \\
\hline
\end{tabular}

The lowest frame is connected to the base of the container having a size of $1000 \mathrm{~mm} \times 495 \mathrm{~mm}$ and a thickness of $40 \mathrm{~mm}$. The outer edge of the base is manufactured to fit the bearings on the shake table, fix to the rotating arm. The box and the table are connected on flight through a crank system.

The inner part of the base is grooved to allow saturation and drainage of the model.

\subsection{Preparation of physical models}

The soil models were reconstituted by air pluviation of dry sand at a target void ratio.

A latex membrane between the soil and the container guarantees water-tightness along the vertical sides. The membrane is fixed to the bottom and the top of the frame stack.

A flexible aluminium mesh was inserted between the soil and the membrane along the short sides of the box. It was connected to the bottom of the stack to improve shear stress transmission at the side boundaries of the soil layer during shaking. 
During soil pluviation the models were instrumented with miniaturised accelerometers and with pore pressure transducers deployed at several depths as shown in Figures 3 (model M1_S2) and 5 (model M1_S3).

Displacement transducers (potentiometers) were located at the ground surface. In this way triggering of the liquefaction was detected and post liquefaction settlements were evaluated. After deposition, the soil layer was saturated with a pore fluid with scaled viscosity. A hydraulic gradient was imposed between the bottom and the top of the layer using a vacuum system. The achievement of complete saturation was controlled by measuring the volume of fluid accumulating in the box and comparing it with the volume of voids.

\subsection{Input ground motions}

The time histories of acceleration were obtained through a two-step procedure aimed at reproducing the expected ground motion at a depth of $15 \mathrm{~m}$ below the ground surface at the prototype scale (Özcebe et al. - unpub.). These records were then applied at the base of the physical models.

In the first step, spectrum-compatible rock outcrop motions were selected from accredited international strong-motion databases for return periods of 475 , 975, and 2475 years. These records were then deconvolved at the roof of seismic bedrock (in Pieve di Cento) and propagated through a soil profile in linear-equivalent ground response analyses up to a depth of $15 \mathrm{~m}$ below the ground surface. Finally, the ground motions were applied at the base of the centrifuge model after appropriate time scaling.

The geotechnical model required to perform ground response analysis at Pieve di Cento was defined based on the available geotechnical data at the site.

Among the 21 calculated time series, 3 were finally selected whose main characteristics are shown in Table 2 . The signals were lastly corrected to take into account the transfer function of the dynamic actuator.

Each model was excited using 3 input motions. A time sufficient to the complete dissipation of the excess pore pressure was allowed between the subsequent application of the various ground motions.

Table 2. Main features of input ground motions

\begin{tabular}{cccccccc}
\hline $\begin{array}{c}\mathrm{T}_{\mathrm{r}} \\
\text { (years) }\end{array}$ & $\begin{array}{c}\mathrm{ID} \\
(-)\end{array}$ & $\begin{array}{c}\mathrm{M}_{\mathrm{W}} \\
(-)\end{array}$ & $\begin{array}{c}\mathrm{R}_{\mathrm{ep}} \\
(\mathrm{km})\end{array}$ & $\begin{array}{c}\mathrm{S}_{\mathrm{F}} \\
(-)\end{array}$ & $\begin{array}{c}\text { Source file } \\
(-)\end{array}$ & $\begin{array}{c}\text { Model } \\
\text { PGA }(\mathrm{g})\end{array}$ & $\begin{array}{c}\mathrm{f}_{\mathrm{p}} \\
(\mathrm{Hz})\end{array}$ \\
\hline 475 & GM17 & 6.1 & 97.0 & 1.65 & $\begin{array}{c}\text { KiKnet } \\
\text { EW2 }\end{array}$ & 5.0 & 500 \\
975 & GM23 & 5.9 & 10.1 & 2.39 & $\begin{array}{c}\text { ESM } \\
\text { ACC }\end{array}$ & 5.3 & 200 \\
2475 & GM34 & 6.9 & 28.6 & 0.59 & $\begin{array}{c}\text { NGA } \\
\text { AT2 }\end{array}$ & 11.3 & 125 \\
\hline
\end{tabular}

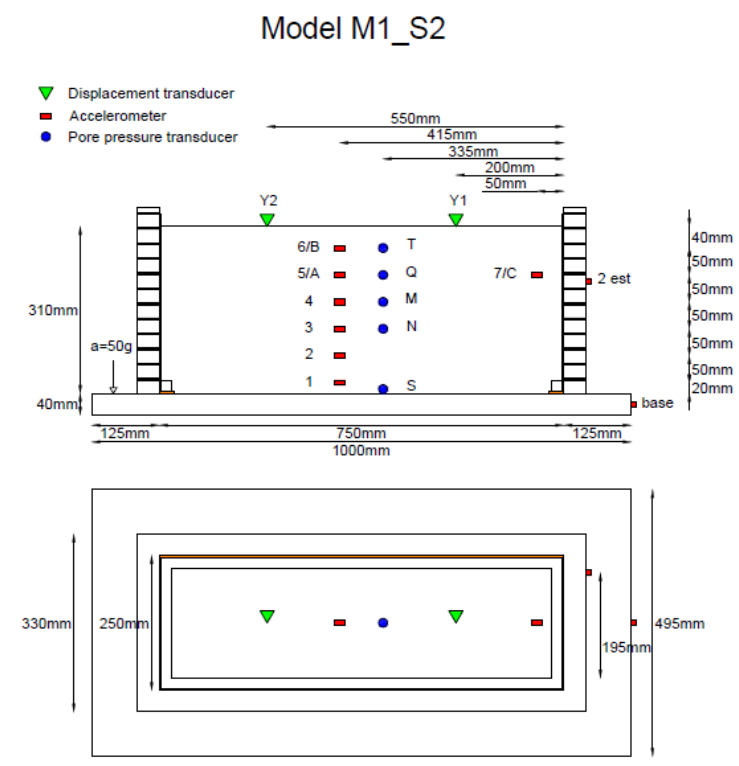

Figure 3. Instrumentation placed in Model M1_S2 to monitor the response of the physical model to ground shaking.

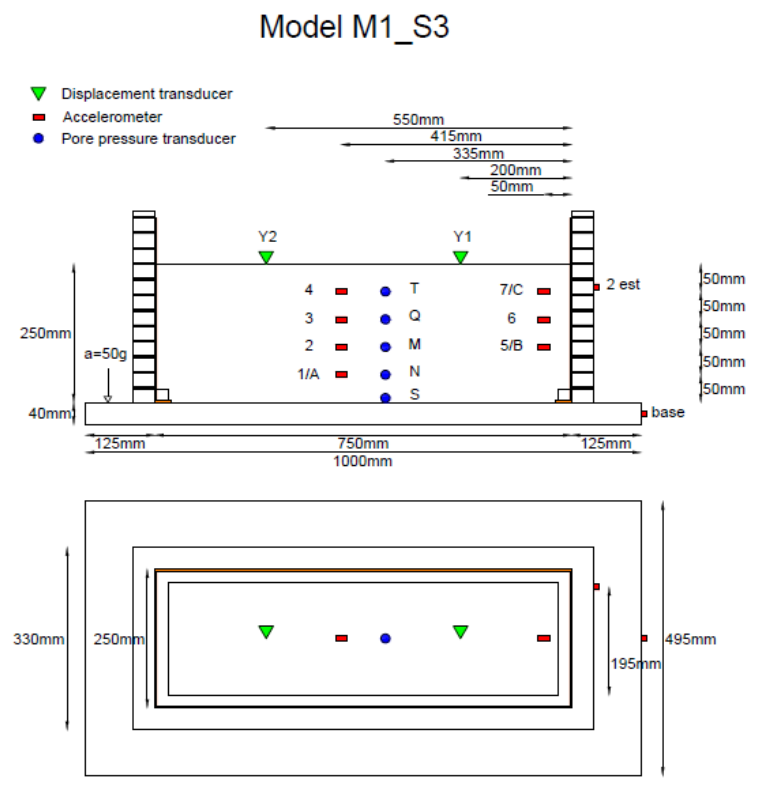

Figure 4. Instrumentation placed in Model M1_S3 to monitor the response of the physical model to ground shaking.

\section{RESULTS}

A selection of results of the tests is shown in this section.

In Figures 5 and 6 the acceleration time history recorded at the base and corresponding to the input signals, is plotted together with the pore pressure ratio $\mathrm{R}_{\mathrm{u}}$, as calculated from the recorded excess pore pressure at the top of the PPT array (T), in models M1_S2 and M1_S3 respectively and for the 3 ground motions. The results clearly show that the accumulation of pore pressures is larger for ground motion of larger amplitude. On the other hand, this accumulation drops during the last run of the series due to sand densification. 


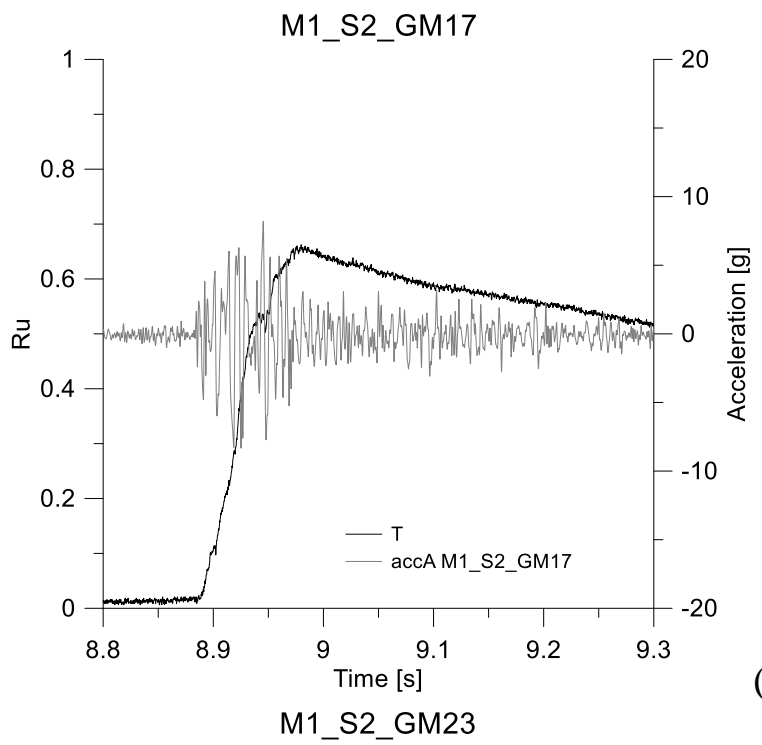

(a)

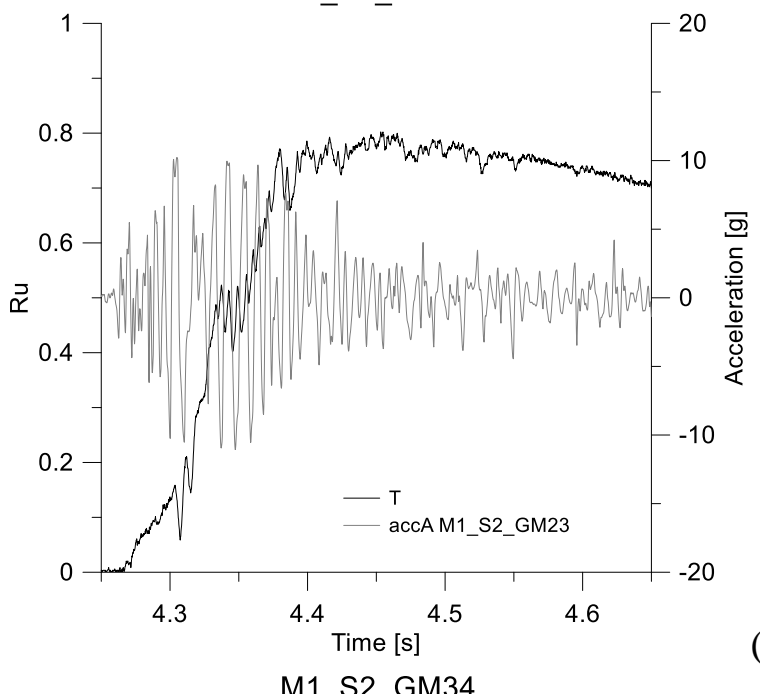

(b)

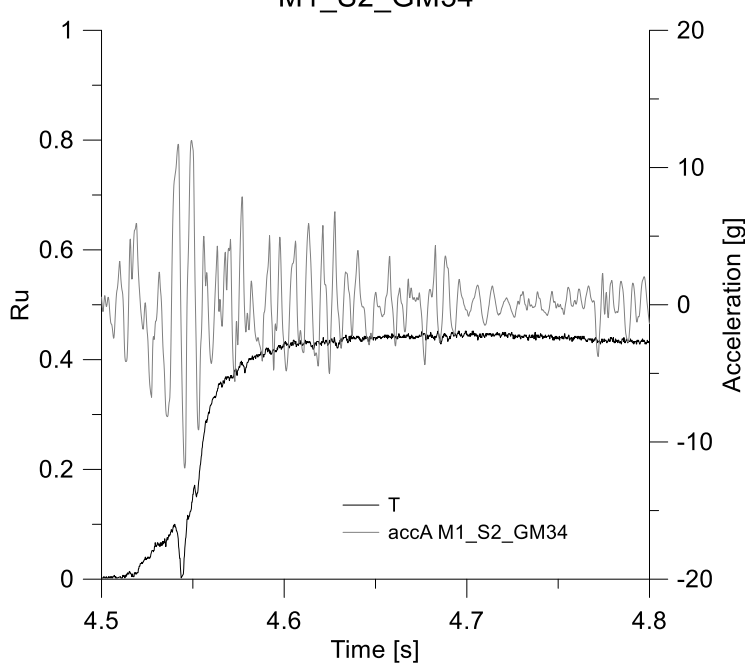

Figure 5. Acceleration time histories at the base and excess pore pressure build-up $(\mathrm{T})$ recorded during the excitation of the shake table in Model M1_S2 for 3 ground motions (model scale)

However, it should be remarked that liquefaction (i.e. $R_{u}=1$ ) was never achieved during the tests.

Figures 7 and 8 illustrate similar plots of the accumulation of settlement at the ground surface, as measured by $\mathrm{Y} 1$ and $\mathrm{Y} 2$ potentiometers. In most cases, the displacements measured by the two transducers are the same at the end of the shaking.

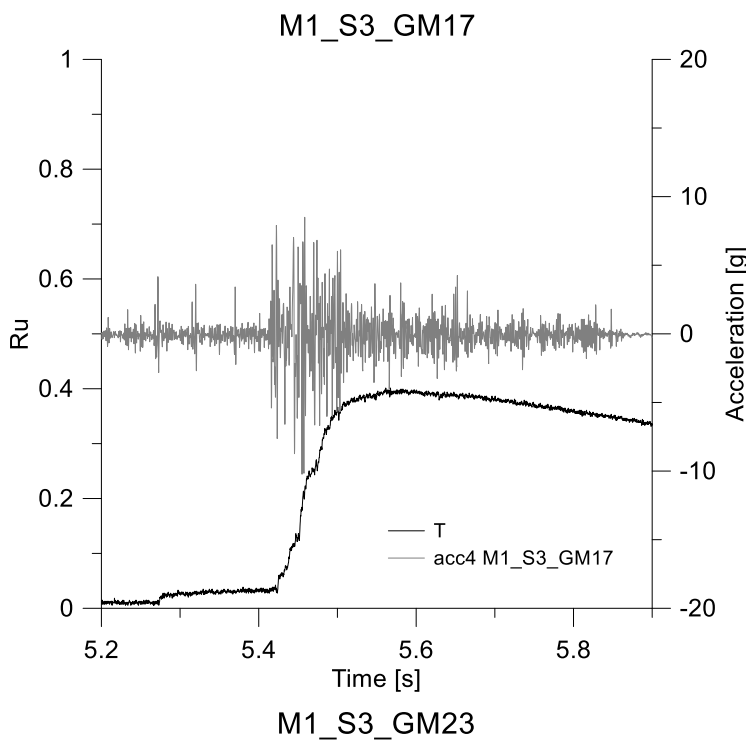

(a)

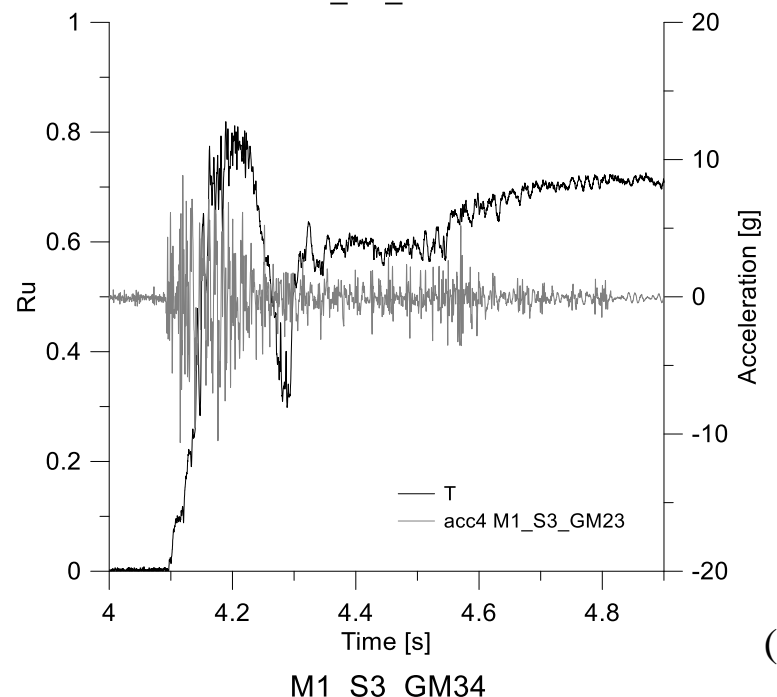

(b)

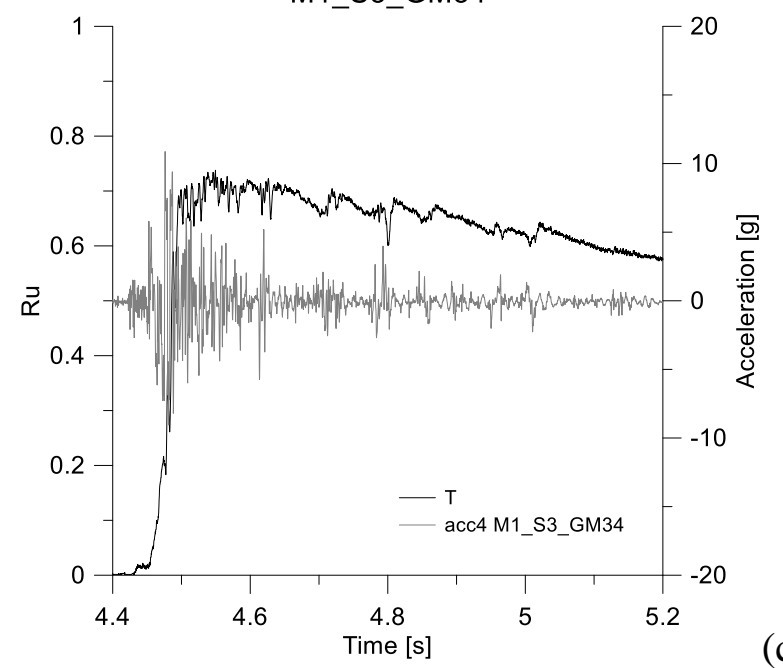

Figure 6. Acceleration time histories at the base and excess pore pressure build-up $(\mathrm{T})$ recorded during the excitation of the shake table in Model M1_S3 for 3 ground motions (model scale)

This indicates a uniform settlement distribution along the ground surface.

Permanent settlement after complete dissipation of excess pore pressure indicates densification of the sandy layer after ground shaking (post-seismic settlement). 


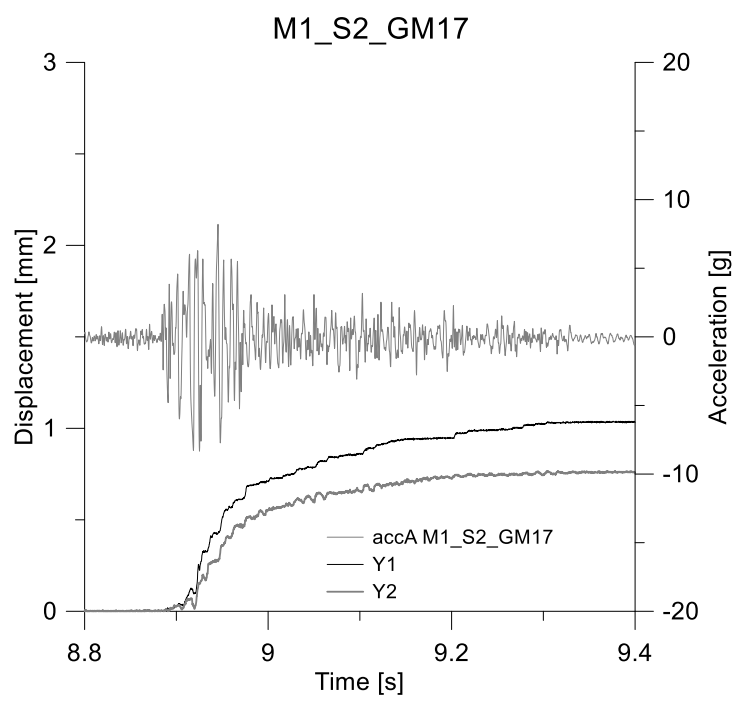

(a)

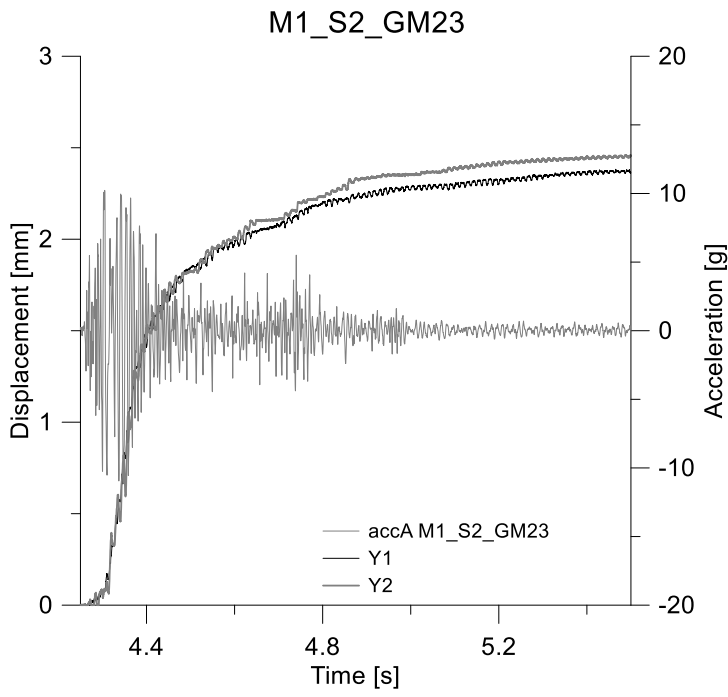

(b)

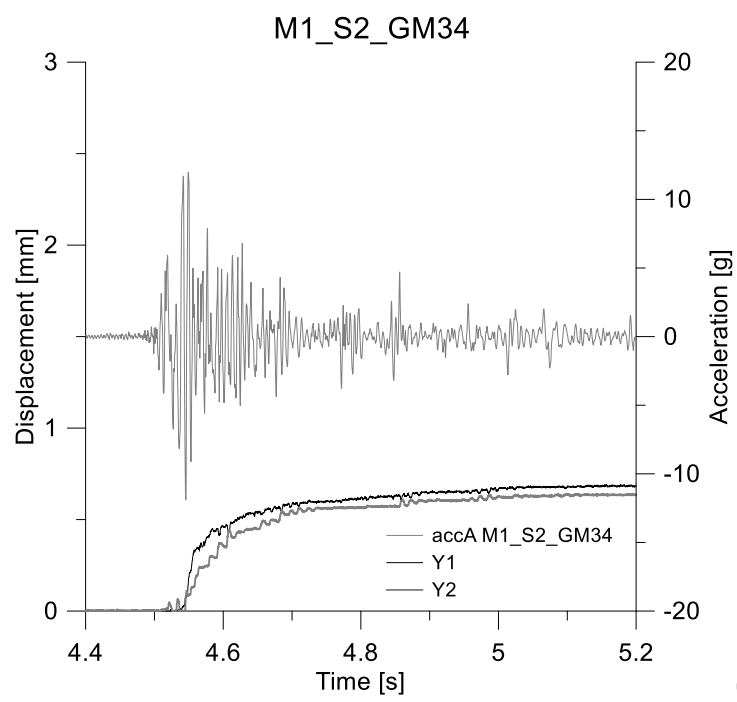

(c)

Figure 7. Acceleration time histories within the soil (A) and measured settlement at surface $(\mathrm{Y} 1, \mathrm{Y} 2)$ recorded during three shakings in Model M1_S2 for 3 ground motions.

Figure 9 shows a comparison of acceleration time histories recorded along the frame (2est) and within the sandy layer (A) during the 3 excitations of Model M1_S2 (Fig. 10a, b and c). The good agreement among the time histories recorded at the same

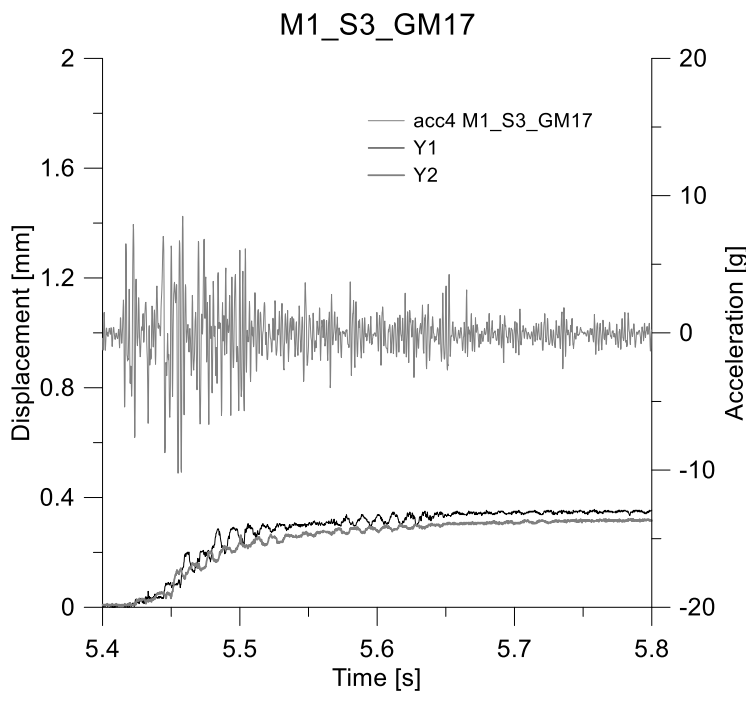

(a)

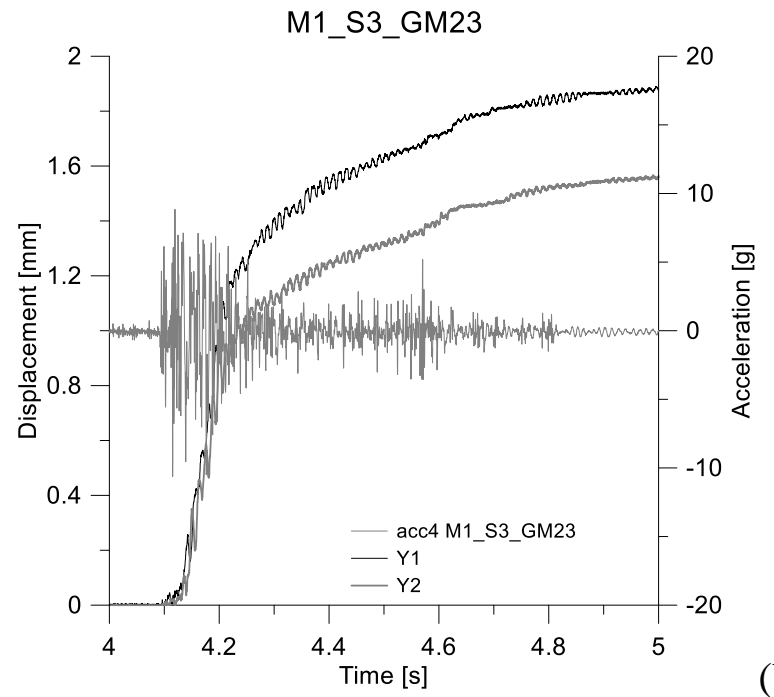

(b)

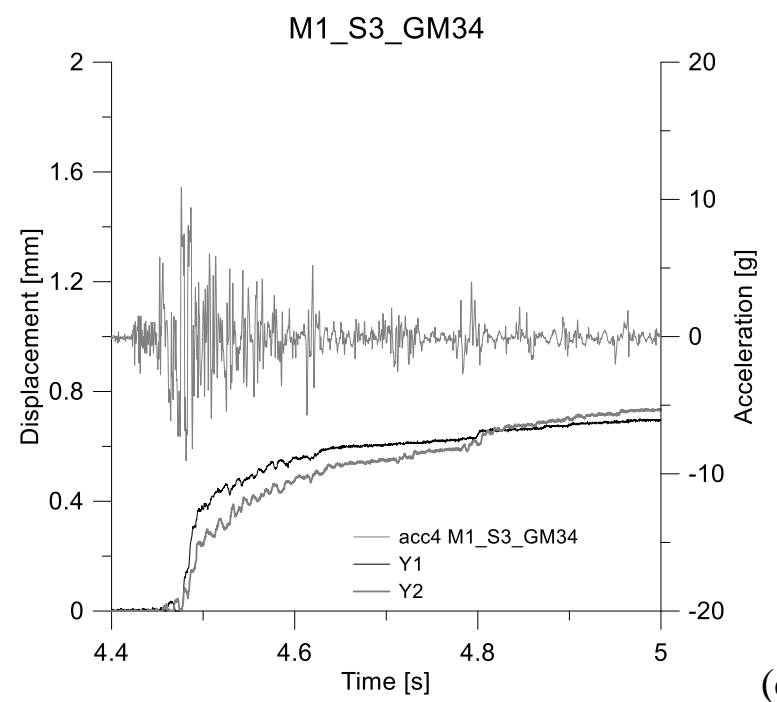

(c)

Figure 8. Acceleration time histories within the soil (4) and measured settlement at surface $(\mathrm{Y} 1, \mathrm{Y} 2)$ recorded during three shakings in Model M1_S3 for 3 ground motions.

elevation along the boundary and within the domain indicates that the ESB container is performing well in mitigating the boundary effects. Only a small amplification of motion is observed towards the end of the inner recorded signal. 


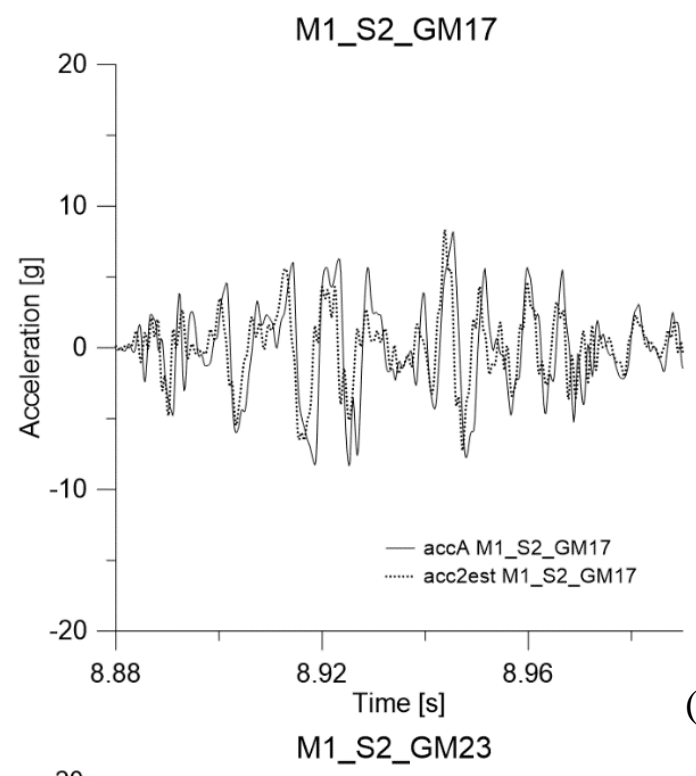

(a)
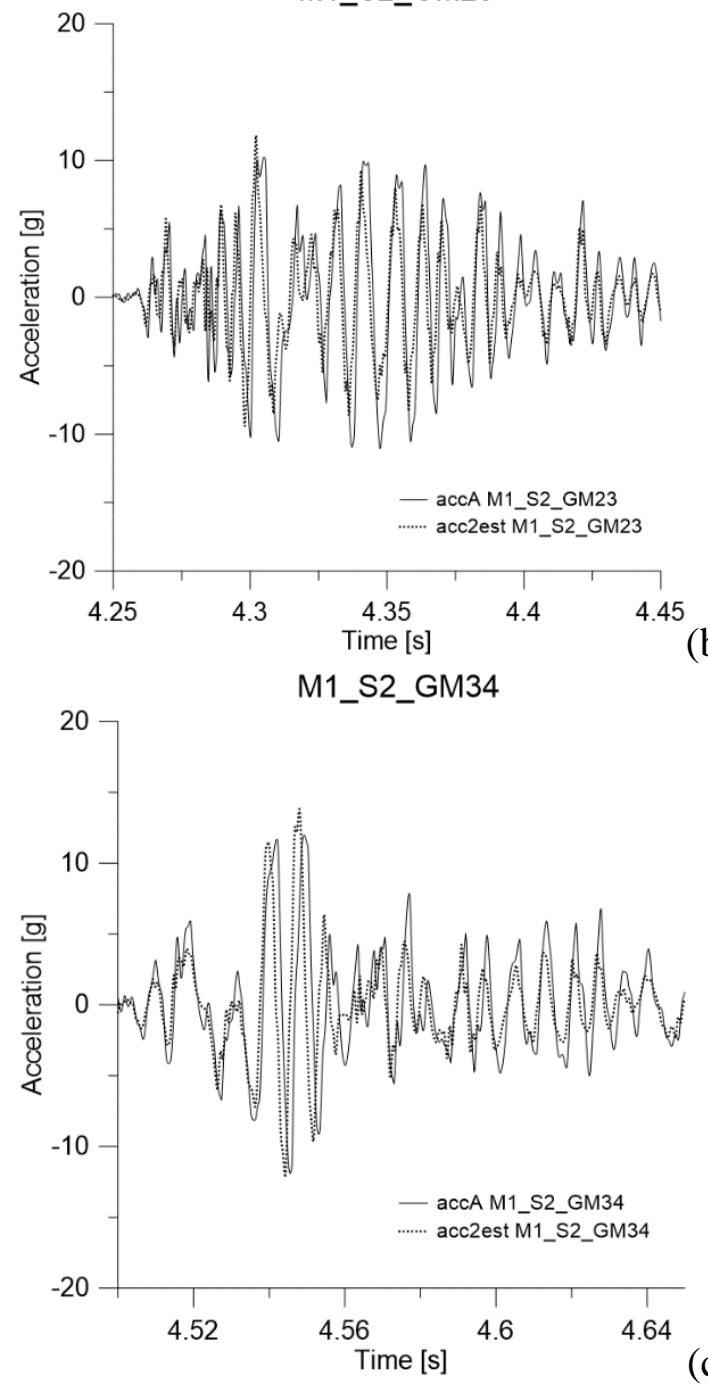

Figure 9. Acceleration time histories on the frame (2est) and within the sandy layer (A) recorded during the excitation of the shake table in Model M1_S2 for 3 ground motions.

\section{CONCLUDING REMARKS}

This paper discussed the results of centrifuge dynamic tests on two physical model of fully saturated sand. These are preliminary tests of a more comprehensive experimental campaign that is carried out on a natural sand retrieved at a site that experienced liquefaction phenomena during the 2012 Emilia-Romagna earthquake in Northern Italy.

Considering the variability of fine content at the site, two different physical models were prepared: one using the sand retrieved at the site, the other by eliminating the fine content prior to the preparation of the model. The time series that were applied at the base of the model were obtained taking into account the expected seismic hazard at the site where the sand was retrieved. Although the pore pressure build-up was clearly observed during all excitations especially in the model with clean sand, true liquefaction was never achieved in any of these two models. Further tests are currently underway both under free-field conditions and in the presence of a building model.

\section{ACKNOWLEDGEMENTS}

This work has been carried out within the LIQUEFACT project. This project has received funding from the European Union's Horizon 2020 research and innovation programme under grant agreement No 700748. The support of F. Bozzoni (Eucentre), E. Zuccolo and A. Famà (University of Pavia) in defining the geotechnical model and the reference ground motions at Pieve di Cento is gratefully acknowledged by the authors.
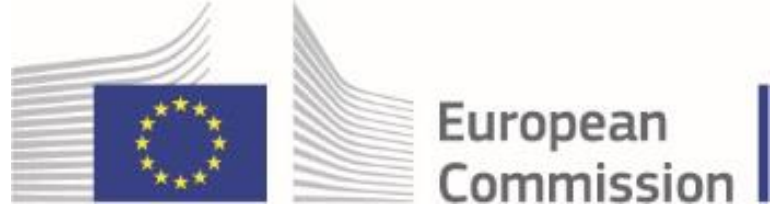

\section{REFERENCES}

Manzari Majid T. et al., 2014. LEAP Projects: Concept and Challenges, Proc. 4th Int. Conf. on Geotechnical Engineering for Disaster mitigation and Rehabilitation (4th GEDMAR), 16-18 September, 2014, Kyoto, Japan.

Mele L. et al. (unpubl.) Report on the comparison of soil response before and after ground treatment at the case study pilot site. Deliverable LIQUEFACT project.

National Academies of Sciences, Engineering, and Medicine, 2016. SoAP in the Assessment of Earthquake-Induced Soil Liquefaction and Its Consequences. Washington, DC: The National Academies Press. https://doi.org/10.17226/23474

Ozcebe AG, Lai CG, E. Zuccolo, Bozzoni F., Famà A. (unpubl.). Definition of shake table motions. WP4 Internal Report. LIQUEFACT project.

Zeng X., Schofield A.N., 1996. Design and performance of an equivalent-shear-beam container for earthquake centrifuge modelling.

Zeghal M. at al. 2014. LEAP: selected data for class C calibrations and class A validations. Proc. 4th Int. Conf. on Geotechnical Engineering for Disaster mitigation and Rehabilitation (4th GEDMAR), 16-18 September, 2014, Kyoto, Japan. 